\title{
Developmental Changes of Tetrahydrobiopterin in Rat and Human Erythrocytes
}

\author{
Kohji Azumagawa, Shuhei Suzuki,* and Makoto Mino \\ Department of Pediatrics, Osaka Medical College, \\ Takatsuki 569, Japan
}

(Received August 22, 1995)

\begin{abstract}
Summary In the present study, we investigated the developmental changes of: (1) plasma and erythrocyte tetrahydrobiopterin (BH4); (2) erythrocyte GTP cyclohydrolase (the rate-limiting enzyme of $\mathrm{BH} 4$ biosynthesis); (3) the permeability of erythrocyte membrane to $\mathrm{BH} 4$; and (4) plasma phenylalanine, both in healthy human subjects and Wistar rats. In vitro experiments demonstrated passive transport of $\mathrm{BH} 4$ into erythrocytes. In humans, BH4 levels as well as the other parameters were fairly consistent across all age groups. In contrast, Wistar rats showed significant developmental changes in erythrocyte $\mathrm{BH} 4$, which were not simply correlated to either GTP cyclohydrolase, permeability to $\mathrm{BH} 4$, or plasma phenylalanine levels. This may suggest the existence of other factors regulating the homeostasis of $\mathrm{BH} 4$, such as $\mathrm{BH} 4$-binding capacity in plasma and/or erythrocytes. These species/age differences in erythrocyte characteristics may influence the pharmacological behavior and clinical efficacy of $\mathrm{BH} 4$ in humans and experimental animals.

Key Words tetrahydrobiopterin, erythrocyte, GTP cyclohydrolase, permeability, phenylalanine, developmental change, human subject, Wistar rat
\end{abstract}

Human mature erythrocytes (RBCs) and plasma have been demonstrated to contain comparable amounts of tetrahydrobiopterin (BH4) $(1,2)$, in spite of a lack of BH4-requiring systems, i.e. the hydroxylation of phenylalanine (3), the biosyntheses of catecholamines (4), serotonin (5) and nitric oxide $(6,7)$ in RBCs. Because of the variety of physiological roles of $\mathrm{BH} 4$, its potential as a therapeutic agent appears to be expanding for many clinical conditions $(8-10)$, in addition to its use as replacement therapy for malignant hyperphenylalaninemia (11), a congenital defect of BH4 biosynthesis.

Recent pharmacological studies have shown that BH4, after being absorbed into the circulation, distributes into RBCs (12) as well as organs such as the liver

\footnotetext{
* To whom correspondence should be addressed.
} 
(13) and brain $(11,14)$. Ponzone et al. (12) have shown that BH4 administration caused an initial increase in plasma $\mathrm{BH} 4$, which was followed by an increase in RBC BH4. Mature RBCs have only trace activity of GTP cyclohydrolase (15), the rate-limiting enzyme in $\mathrm{BH} 4$ biosynthesis (16), while mature $\mathrm{RBCs}$ are rich in the BH4 recycling system, i.e. dihydropteridine reductase (17). Therefore, it could be hypothesized that the majority of $\mathrm{BH} 4$ in peripheral RBCs may be derived from plasma, and that the RBC may play an important role as a source of BH4 in the circulation.

Other studies have demonstrated that plasma and $\mathrm{RBC}$ BH4 are increased by a phenylalanine load in healthy human subjects (18) and in a patient with congenital BH4 deficiency along with high level of plasma phenylalanine (12). Therefore phenylalanine levels are thought to be one of the factors regulating RBC and plasma BH4 levels.

Furthermore, a recent autoradiographic study in rats using radioactive BH4 (13) demonstrated that exogenous BH4 was distributed mainly into liver and kidney, and this distribution changed with development. Developmental changes in RBC BH4 may be an important factor affecting the pharmacological behavior and clinical efficacy of administered BH4. However, little is known about BH4 concentrations in blood, especially $\mathrm{RBC} \mathrm{BH} 4$ concentrations and the permeability of the RBC membrane to BH4.

In this study, the developmental changes in (1) BH4 levels in plasma and RBC, (2) RBC GTP cyclohydrolase activity, (3) RBC membrane permeability to BH4, and (4) plasma phenylalanine levels, both in Wistar rats and healthy human subjects were investigated.

\section{METHODS AND MATERIALS}

Human subjects. Human blood samples were collected from inpatients and outpatients at the Osaka Medical College Hospital, Japan. Cord blood $(n=36)$ was collected from a placental vein immediately after the placenta was delivered. Blood from the products of a complicated pregnancy or premature delivery was excluded. Blood samples from neonates $(n=62)$, infants $(n=24)$, and children $(n=40)$ were from the residua of blood samples drawn for other laboratory tests. Each age group was defined as follows: less than 1 month (neonates, mean age; 27.3 days), 1 month to 12 months (infants, mean age; 5.6 months), and over 1 year to 15 years old (children, mean age; 7.1 years). Adult blood was drawn from healthy volunteers aged from 23 to 37 years (mean age; 28.3 years). We excluded subjects who were diagnosed as having malignant diseases, autoimmune diseases, severe allergies, who were being treated with steroids or other immunomodulating agents, or who had symptoms or laboratory evidence of viral/bacterial infections (e.g. recent febrile episodes, diarrhea, or a positive C-reactive protein). Most blood samples were residual samples from negative screening tests for rare metabolic or endocrine disorders. 
Due to the small amounts of the blood samples, we did not perform GTP cyclohydrolase assay in infant and child RBCs, and permeability tests in cord, infant and child RBCs.

Rat study. Blood from male Wistar rats (Japan SLC Co., Shizuoka, Japan) was obtained by heart puncture under diethyl ether anesthesia. The rats were fed ad libitum, kept in our vivarium, which was maintained at a constant temperature and day-night cycle, for a week before sacrifice. The rats younger than 3 weeks were kept with the mother rats prior to sampling. All the rats in an age group were sacrificed on the same day, and the procedure was performed at the same time of day and at the same place by one person.

Sample preparation. The blood samples were immediately heparinized with ascorbic acid as an antioxidant to a final concentration of $10 \mathrm{mg} / \mathrm{ml}$. The reduced pterins were found to be stable under these conditions at room temperature for at least $4 \mathrm{~h}$ (data not shown). After plasma was separated by centrifugation $(1,000 \times$ $g, 4^{\circ} \mathrm{C}, 15 \mathrm{~min}$ ), RBCs were washed with cold saline and recentrifuged twice. The buffy coat was removed by aspiration as much as possible. The suspension was checked for packed cell volume and reticulocyte count after staining with fresh methylene blue. The reticulocyte counts in the samples analyzed were all negligible $(<0.1 \%)$. No detectable pterins were found to have leaked out during the washing steps. The samples were either prepared immediately for measurements of $\mathrm{BH} 4$ content and permeability of $\mathrm{BH} 4$, or stored at $-80^{\circ} \mathrm{C}$ until GTP cyclohydrolase assays were performed.

Biopterin assay. The BH4 contents were determined by a modified Fukushima's method (19). One milliliter of plasma or RBC suspension (approximately $0.3 \mathrm{ml}$ of packed cells) was oxidized by adding $1 \mathrm{ml}$ of $2 \mathrm{~N}$ trichloroacetic acid, $3 \mathrm{ml}$ of $0.4 \mathrm{~N}$ phosphoric acid, $3 \mathrm{ml}$ of acidic iodine solution $\left(1 \% \mathrm{I}_{2}, 2 \% \mathrm{KI}\right.$ in $\left.1 \mathrm{~N} \mathrm{HCl}\right)$, and $50 \mathrm{pmol}$ of 6 -methylpterin as the internal standard. This oxidized mixture was left in the dark at room temperature for $2 \mathrm{~h}$, and the reaction was stopped by the addition of $0.3 \mathrm{ml}$ of $20 \%$ ascorbic acid. After centrifugation, the supernatant was applied to a column of Dowex $50 \mathrm{~W}-\mathrm{X} 8(0.5 \mathrm{ml}$ bed volume), and the column was washed with $5 \mathrm{ml}$ of water. Pterins were eluted with $4 \mathrm{ml}$ of $1 \mathrm{~N}$ ammonium hydroxide and the eluate was then applied directly onto a Dowex $1 \mathrm{X}-8$ column. After the resin was washed with water, pterins were eluted with $1 \mathrm{~N}$ acetic acid. The eluate was dried in a rotary evaporator. The residue was dissolved in $1 \mathrm{ml}$ of water and $0.2 \mathrm{ml}$ of this solution was applied to an HPLC equipped with a fluorescence detector.

The HPLC system consisted of an LC-5A pump, C-R3B integrator, RF-535 fluorometric detector (Shimadzu, Kyoto, Japan), AS-8010 autoinjector (TOSO, Tokyo, Japan), G-1300 degasser (Irika, Kyoto, Japan), and a Partisil 10-ODS HPLC column $(4.6 \times 250 \mathrm{~mm})$ with a Universal guard cartridge column (Whatman, NJ, USA). Five percent methanol in distilled water (flow rate: $0.8 \mathrm{ml} / \mathrm{min}$ ) was used as a mobile phase for determination of biopterin. The detector was set at $350 \mathrm{~nm}$ for excitation and $440 \mathrm{~nm}$ for emission.

Vol. 41, No. 6, 1995 
The recovery of $\mathrm{BH} 4$ and the internal standard was no less than $80 \%$ in this assay.

GTP cyclohydrolase in RBC. GTP cyclohydrolase activity in blood samples was measured in both humans and Wistar rats of various ages. Enzyme activity was assayed by a modified method of Duch et al. (1). Washed RBCs were hemolyzed by ultrasonication in $0.1 \mathrm{M}$ Tris- $\mathrm{HCl}$ buffer $(\mathrm{pH} 7.8)$ containing $0.3 \mathrm{M} \mathrm{KCl}, 2.5 \mathrm{~mm}$ EDTA and $0.25 \mathrm{~mm}$ phenylmethylsulfonylfluoride. The supernatant was immediately concentrated using Centriflo ${ }^{\circledR}$ ultrafiltration membrane cones (Amicon, Tokyo, Japan), and applied to a PD-10 column (Pharmacia-LKB, Uppsala, Sweden) equilibrated with the above buffer. After the first $2.5 \mathrm{ml}$ eluate was discarded, the next 3.0-ml fraction was collected for enzyme assay and simultaneous protein concentration (20). All steps were carried out at $4{ }^{\circ} \mathrm{C}$. The reaction mixture $(500 \mu \mathrm{l})$ consisted of $0.1 \mathrm{M}$ Tris- $\mathrm{HCl}$ buffer $(\mathrm{pH} 7.8), 1 \mathrm{mM}$ GTP and the enzyme. The incubation was performed in the dark at $37^{\circ} \mathrm{C}$ for $2 \mathrm{~h}$, and the enzyme reaction was stopped by adding $50 \mu \mathrm{l}$ of acidic iodine solution. The mixture was left in the dark at room temperature for $1 \mathrm{~h}$ for oxidation, which was stopped by adding $50 \mu \mathrm{l}$ of $20 \%$ ascorbic acid. After adding $100 \mu \mathrm{l}$ of $1 \mathrm{~N}$ acetic acid and $50 \mu \mathrm{l}$ of 2 $\mathrm{N}$ trichloroacetic acid, the precipitate was removed by centrifugation and trichloroacetic acid remaining in the supernatant was removed by extracting with water-saturated diethyl ether. The extracted supernatant was applied to an HPLC to quantitate the neopterin produced as pmol of neopterin per hour per mg protein.

The HPLC was equipped with a Cosmosil $5 \mathrm{C}_{18}$ column $(4.6 \times 250 \mathrm{~mm})$ and a Cosmosil $5 \mathrm{C}_{18}$ guard column $(4.6 \times 50 \mathrm{~mm}$, Nacalai Tesque, Kyoto, Japan $)$ for the detection of neopterin. The mobile phase was $10 \mathrm{~mm}$ sodium phosphate buffer $(\mathrm{pH}$ 7.0) containing $1 \mathrm{mM}$ EDTA run at a flow rate of $0.8 \mathrm{ml} / \mathrm{min}$. The fluorescence detector was set at $350 \mathrm{~nm}$ for excitation and $440 \mathrm{~nm}$ for emission.

Permeability of $\mathrm{RBCs}$ to $\mathrm{BH} 4$. The in vitro influx and efflux of $\mathrm{BH} 4$ across the RBC membrane were determined as follows: RBCs washed with saline were suspended in, and adjusted to $20 \%$ cell suspension, in phosphate-buffered saline ( $\mathrm{pH}$ 7.4) with $1 \mathrm{mg} / \mathrm{ml}$ ascorbic acid and a given concentration of $\mathrm{BH} 4$. This medium was thoroughly degassed and saturated with $\mathrm{N}_{2}$ gas just prior to use. Immediately after the scheduled incubation time at $37^{\circ} \mathrm{C}$ in dark, RBCs were separated by brief centrifugation at $4{ }^{\circ} \mathrm{C}$, followed by two successive washings with cold saline. Then, BH4 content was determined as described above.

The initial linear increase of intracellular $\mathrm{BH} 4$ was determined after $15 \mathrm{~min}$ of incubation with various concentrations of $\mathrm{BH} 4$ in the medium. Since the correlation was linear between $\mathrm{BH} 4$ concentrations in the medium and the initial increase rate of intracellular BH4 (Fig. 1B), the permeability of the RBC membrane was calculated according to the following first-order equation: $[\mathrm{V}]=\mathrm{k} \times[\mathrm{A}]$, where $[\mathrm{V}]$ is the initial rate of increase in $\mathrm{RBC} \mathrm{BH} 4$ concentration and [A] is the $\mathrm{BH} 4$ concentration gradient between medium and $\mathrm{RBC}$. The " $\mathrm{k}$ " is expressed in $\mathrm{s}^{-1}$.

To determine BH4 efflux via the RBC membrane, RBCs with elevated BH4 concentrations were first produced by incubating RBCs with buffer containing 50 
$\mu \mathrm{M} \mathrm{BH} 4$ for $20 \mathrm{~min}$ at $37^{\circ} \mathrm{C}$ in the dark. After incubation, RBCs were washed twice with cold saline. The RBCs with a large amount of $\mathrm{BH} 4$ were suspended in the medium without $\mathrm{BH} 4$, and the packed cell volume was adjusted to $5 \%$ of the suspension. The reaction was started after $8 \mathrm{~min}$ preincubation at $37^{\circ} \mathrm{C}$, and the incubation was stopped by prompt cooling and quick centrifugation at the scheduled times. The RBC BH4 content was then determined after the RBCs were washed twice with cold saline. The decrease in intracellular BH4 was also linear up to 20 min of incubation after the preincubation. The permeability coefficient " $\mathrm{k}$ " was calculated similarly to the influx rate.

Phenylalanine levels in human and rat plasma. After plasma was deproteinized with trichloroacetic acid, the supernatant was analyzed for phenylalanine content with a L-8500 High-performance amino acid analyzer (Hitachi, Osaka, Japan).

Statistical analysis. All values (see tables) are expressed as $\mathbf{M} \pm \mathbf{S D}$. Statistical differences were examined by one-way analysis of variance (ANOVA) using a Fisher PLSD to determine significance.

Materials and equipments. Chemicals were obtained as follows: neopterin, biopterin, 6-methylpterin and alkaline phosphatase (bovine intestine) from Sigma (St. Louis, MO, USA); Dowex 50W-X8 ( $\mathrm{H}^{+}$form, 200-400 mesh) and Dowex 1-X 8 ( $\mathrm{Cl}^{-}$form, 200-400 mesh) from Dow Chemical Co. (Midland, MI. USA); GTP from Yamasa Co. (Chiba, Japan). Other chemicals were all analytical grade. BH4 was provided by Suntory Institute of Biomedical Research (Osaka, Japan).

\section{RESULTS}

\section{Human plasma and $\mathrm{RBC} B \mathrm{BH}$ contents}

Both plasma and RBC BH4 levels were similar across all age groups (Table 1). Only children's plasma and RBC BH4 were significantly higher than in the neonate and infant groups, as has been reported previously (21). RBC BH4 levels did not significantly differ from those in plasma (Table 1).

Table 1. Plasma and RBC BH4 concentrations in human subjects.

\begin{tabular}{lcc}
\hline \multirow{2}{*}{ Group } & \multicolumn{2}{c}{ BH4 $(\mathrm{pmol} / \mathrm{ml})$} \\
\cline { 2 - 3 } & \multicolumn{1}{c}{ Plasma } & RBC \\
\hline Cord $(n=36)$ & $14.0 \pm 5.5$ & $12.2 \pm 5.7$ \\
Neonate $(n=62)$ & $11.4 \pm 3.6$ & $10.1 \pm 3.8$ \\
Infant $(n=24)$ & $11.5 \pm 4.7$ & $11.1 \pm 6.6$ \\
Child $(n=40)$ & $16.9 \pm 10.6^{*}$ & $14.5 \pm 8.4^{* *}$ \\
Adult $(n=20)$ & $14.8 \pm 6.1$ & $12.5 \pm 7.1$ \\
\hline
\end{tabular}

The data are expressed as the $\mathbf{M} \pm \mathbf{S D}$. * Significantly different from neonate and infant by ANOVA using Fisher PLSD (at 99.5\%). ** Significantly different from neonate by ANOVA using Fisher PLSD (at 99.5\%). 


\section{$B H 4$ contents in Wistar rat plasma and $R B C$}

BH4 levels in Wistar rat plasma and RBCs were higher than those of human subjects (Table 2). There were no significant differences in plasma BH4 concentrations among the rat groups, except that plasma $\mathrm{BH} 4$ were significantly greater in 1week-old rats than in 3-week-old rats. Regarding RBC, the BH4 level in 1-week-old rats was significantly greater than those in 4-, 6-, and 10-week-old rats. The developmental change was more prominent in RBCs than in plasma.

\section{$R B C$-to-plasma BH4 concentration ratios}

The RBC-to-plasma ratio of BH4 in humans did not differ among the various age groups. However, the RBC-to-plasma ratio of $\mathrm{BH} 4$ in rats was very high compared with those in humans, and the ratio in rats was also found to be very much greater in the younger age groups (Table 3 ).

GTP cyclohydrolase activity in human and rat RBCs

In human RBC, GTP cyclohydrolase activity was slightly, but not significantly

Table 2. Plasma and RBC BH4 concentrations in Wistar rats.

\begin{tabular}{ccc}
\hline \multirow{2}{*}{ Age } & \multicolumn{2}{c}{ BH4 $(\mathrm{pmol} / \mathrm{ml})$} \\
\cline { 2 - 3 } & Plasma & RBC \\
\hline 1 week $(n=8)$ & $211 \pm 52^{*}$ & $3,660 \pm 2,070^{* *}$ \\
3 week $(n=5)$ & $141 \pm 41$ & $1,770 \pm 489$ \\
4 week $(n=5)$ & $152 \pm 15$ & $810 \pm 316$ \\
6 week $(n=5)$ & $160 \pm 27$ & $645 \pm 107$ \\
10 week $(n=3)$ & $196 \pm 59$ & $933 \pm 198$ \\
\hline
\end{tabular}

The data are expressed as the $\mathbf{M} \pm \mathrm{SD}$. * Significantly different from 3-week-old rats by ANOVA using Fisher PLSD (at 99.17\%). ** Significantly different from 4-, 6and 10-week-old rats by ANOVA using Fisher PLSD (at 99.17\%).

Table 3. RBC-to-plasma BH4 concentration ratios $(\mathrm{R} / \mathrm{P})$ in human subjects and Wistar rats.

\begin{tabular}{lcccc}
\hline \multicolumn{2}{c}{ Human } & & \multicolumn{2}{c}{ Wistar rat } \\
\cline { 1 - 2 } \cline { 5 - 5 } \multicolumn{1}{c}{ Group } & $\mathrm{R} / \mathrm{P}$ of $\mathrm{BH} 4$ & & Age & $\mathrm{R} / \mathrm{P}$ of BH4 \\
\hline Cord $(n=35)$ & $0.92 \pm 0.42$ & & 1 week $(n=7)$ & $15.6 \pm 4.5^{*}$ \\
Neonate $(n=62)$ & $0.98 \pm 0.46$ & & 3 week $(n=5)$ & $12.8 \pm 4.3^{* *}$ \\
Infant $(n=22)$ & $1.04 \pm 0.62$ & & 4 week $(n=5)$ & $5.4 \pm 2.1$ \\
Child $(n=45)$ & $1.05 \pm 0.55$ & & 6 week $(n=5)$ & $4.0 \pm 0.6$ \\
Adult $(n=20)$ & $1.01 \pm 0.65$ & & 10 week $(n=3)$ & $4.8 \pm 0.6$ \\
\hline
\end{tabular}

The data are expressed as the $\mathrm{M} \pm \mathrm{SD}$. * Significantly different from 4- and 6-weekold rats by ANOVA using Fisher PLSD (at 99.17\%). ** Significantly different from 6-week-old rats by ANOVA using Fisher PLSD (at 99.17\%). 
higher in neonates than in adults or cord blood. In addition, the GTP cyclohydrolase activity was not significantly correlated with the RBC BH4 content ( $r=$ 0.147 ). In rat $\mathrm{RBCs}$, the enzyme activity was higher in the younger groups. This change was consistent with the developmental change in rat RBC BH4 concentrations, except that the 1-week-old rats had low RBC GTP cyclohydrolase activity, despite having the highest BH4 levels in both RBCs and plasma (Table 4).

\section{Permeability of human and rat $\mathrm{RBCs}$ to $\mathrm{BH} 4$}

There was a linear increase in intracellular BH4 during the 60-min incubation of RBCs with $5 \mu \mathrm{M} \mathrm{BH} 4$ (Fig. 1A). The increasing rate of cellular BH4 was also linearly correlated with the BH4 concentration in the medium (Fig. 1B). As shown in Table 5, although both the influx and efflux were somewhat greater in neonatal RBCs than in adult RBCs, the difference in permeability was not statistically significant. Rat RBCs were more permeable in vitro to BH4 than human RBCs. Furthermore, the efflux coefficient in rats was several fold greater than the influx coefficient in all age groups. The difference in permeability among the groups of rats did not reach significance.

\section{Phenylanine levels}

Human plasma phenylalanine levels in neonates were slightly and significantly lower than those in the other age groups. In the rats, phenylalanine levels did not differ significantly between age groups (Table 6). Phenylalanine concentrations were not correlated significantly with either plasma or RBC BH4 levels in any age group, in either humans or Wistar rats (data not shown).

\section{DISCUSSION}

These results demonstrate that human RBCs and plasma contain almost the same amounts of BH4 in all age groups (Tables 1,3). This finding may be

Table 4. RBC GTP cyclohydrolase activity in humans and Wistar rats.

\begin{tabular}{lcrcc}
\hline \multicolumn{2}{c}{ Human } & & \multicolumn{2}{c}{ Wistar rat } \\
\cline { 1 - 2 } \cline { 5 - 5 } Group & GTP cyclohydrolase & & Age & GTP cyclohydrolase \\
\hline Cord $(n=6)$ & $0.45 \pm 0.24$ & & 1 week $(n=5)$ & $1.6 \pm 0.9$ \\
Neonate $(n=7)$ & $0.67 \pm 0.31$ & & 3 week $(n=5)$ & $3.1 \pm 1.2^{*}$ \\
Adult $(n=9)$ & $0.57 \pm 0.09$ & & 4 week $(n=6)$ & $2.4 \pm 0.6^{* *}$ \\
& & & 6 week $(n=5)$ & $0.8 \pm 0.1$ \\
& & & 10 week $(n=3)$ & $1.3 \pm 0.6$ \\
\hline
\end{tabular}

The enzyme activity is expressed in $\mathrm{pmol} / \mathrm{h} \cdot \mathrm{mg}$ protein, and the values are $\mathbf{M} \pm \mathrm{SD}$.

* Significantly different from 6- and 10-week-old rats by ANOVA using Fisher PLSD (at 99.5\%). ** Significantly different from 6-week-old rats by ANOVA using Fisher PLSD (at 99.5\%). 

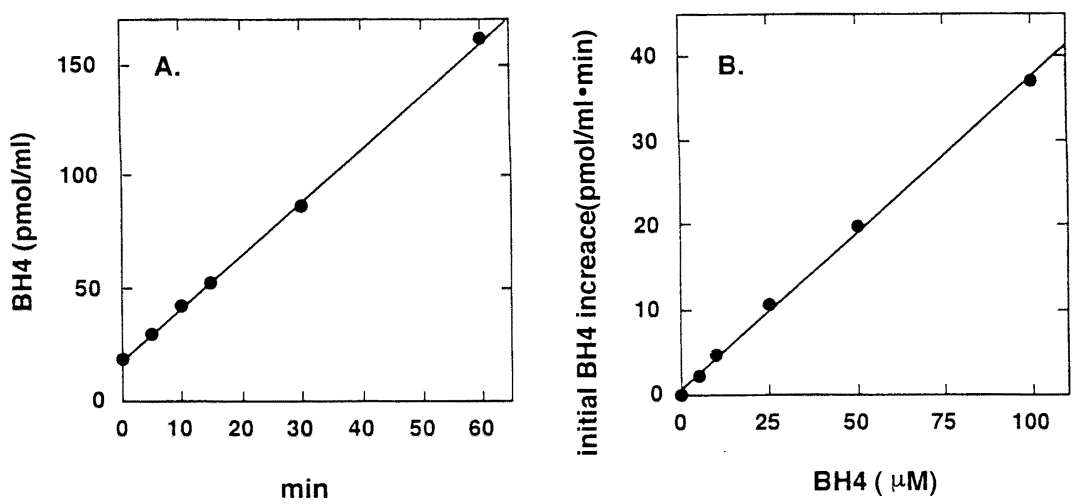

Fig. 1. (A): Time-dependent increase of BH4 level in RBC when adult RBC was incubated at $37^{\circ} \mathrm{C}$ with $5 \mu \mathrm{M}$ of $\mathrm{BH} 4$ in the medium (phosphate-buffered saline). The intracellular BH4 increased linearly at least until $60 \mathrm{~min}$ under the conditions described in "Methods and Materials." (B): The relationship between the initial increase rate of $\mathrm{BH} 4$ in $\mathrm{RBC}(\mathrm{pmol} / \mathrm{ml} \cdot \mathrm{min})$ and the $\mathrm{BH} 4$ concentration of the medium. Washed RBCs from an adult volunteer were incubated at $37^{\circ} \mathrm{C}$ with various concentrations of BH4 in the medium $(0-100 \mu \mathrm{M})$. The first-degree relationship was demonstrated between the $\mathrm{BH} 4$ concentration in the medium and the initial increase rate of $\mathrm{BH} 4$ in $\mathrm{RBC}$, indicating the passive transport of BH4 into RBCs.

Table 5. In vitro BH4 permeability in human and Wistar rat RBCs.

\begin{tabular}{|c|c|c|c|c|c|}
\hline \multicolumn{3}{|c|}{ Human } & \multicolumn{3}{|c|}{ Wistar rat } \\
\hline \multirow{2}{*}{ Group } & \multicolumn{2}{|c|}{$\mathrm{k}\left(\mathrm{s}^{-1}\right)$} & \multirow{2}{*}{ Age } & \multicolumn{2}{|c|}{$\mathrm{k}\left(\mathrm{s}^{-1}\right)$} \\
\hline & Influx & Efflux & & Influx & Efflux \\
\hline Neonate $(n=9)$ & $0.35 \pm 0.09$ & $0.28 \pm 0.04$ & 1 week $(n=5)$ & $0.78 \pm 0.34$ & $4.9 \pm 0.91$ \\
\hline & & & 4 week $(n=5)$ & $1.1 \pm 0.12$ & $5.3 \pm 0.23 *$ \\
\hline Adult $(n=12)$ & $0.19 \pm 0.04$ & $0.16 \pm 0.07$ & 9 week $(n=5)$ & $1.0 \pm 0.06$ & $4.0 \pm 0.26$ \\
\hline
\end{tabular}

The coefficient " $\mathrm{k}$ " $\left(\mathrm{s}^{-1}\right)$ was calculated by the equation; $[\mathrm{V}]=\mathrm{k} \times[\mathrm{A}]$, where $[\mathrm{V}]$ is the initial increase or decrease rate of $\mathrm{BH} 4$ concentration in packed $\mathrm{RBC}$ (pmol/ $\mathrm{ml} / \mathrm{s}$ ), and $[\mathrm{A}]$ is the concentration gradient between the medium and $\mathrm{RBC}(\mathrm{pmol} /$ $\mathrm{ml}$ ). The data are expressed as the $\mathbf{M} \pm \mathrm{SD}$. ${ }^{*}$ Significantly different from 9-week-old rats by ANOVA using Fisher PLSD (at 98.33\%).

compatible with the hypothesis that most of the BH4 in RBCs is derived from plasma by passive diffusion (Fig. 1), and that the RBC may act as a BH4 reservoir in the circulation, especially for exogenous $\mathrm{BH} 4$ given as a therapeutic agent (12). In addition, with respect to the flux rate of $\mathrm{BH} 4$ via the $\mathrm{RBC}$ membrane, the influx did not differ from the efflux either in neonatal or adult RBCs. This finding is also 
Table 6. Plasma phenylalanine concentrations.

\begin{tabular}{lcccc}
\hline \multicolumn{2}{c}{ Human } & & \multicolumn{2}{c}{ Wistar rat } \\
\cline { 1 - 2 } \cline { 5 - 5 } \multicolumn{1}{c}{ Group } & $\begin{array}{c}\text { Phyenylalanine } \\
(\mathrm{nmol} / \mathrm{ml})\end{array}$ & & Age & $\begin{array}{c}\text { Phyenylalanine } \\
(\mathrm{nmol} / \mathrm{ml})\end{array}$ \\
\hline Cord $(n=14)$ & $67.1 \pm 9.3$ & & 1 week $(n=5)$ & $63.0 \pm 4.4$ \\
Neonate $(n=24)$ & $43.2 \pm 10.0^{*}$ & & 3 week $(n=5)$ & $62.7 \pm 6.5$ \\
Infant $(n=10)$ & $59.8 \pm 19.4$ & & 4 week $(n=6)$ & $60.1 \pm 13.6$ \\
Child $(n=8)$ & $55.0 \pm 5.3$ & & 6 week $(n=5)$ & $67.6 \pm 4.4$ \\
Adult $(n=14)$ & $57.4 \pm 10.6$ & & 10 week $(n=3)$ & $65.3 \pm 1.8$ \\
\hline
\end{tabular}

* Significantly different from cord, infant and adult by ANOVA using Fisher PLSD (at $99.5 \%$ ).

consistent with the hypothesis that the $\mathrm{RBC}$ may act as a simple container for $\mathrm{BH}$ 4 in the circulation.

On the other hand, Wistar rats were demonstrated to be quite different from humans in terms of BH4 cellular disposition, and developmental changes. Rat RBC BH4 levels were considerably higher than that of plasma in all age groups (Tables 2, 3). The fact that rat RBCs were richer in GTP cyclohydrolase activity than human RBCs (Table 4) may explain the gap between rat RBC and plasma BH4 levels. Regarding the developmental changes, however, there was no correlation between the enzyme activity and RBC BH4 content, especially in the RBCs of 1week-old rats, which had the highest $\mathrm{BH} 4$ content among the age groups, yet had lower GTP cyclohydrolase activity than did the 3-and 4-week-old rats. This indicates that enhanced in situ biosynthesis of $\mathrm{BH} 4$ may not be responsible for the higher neonatal Wistar rat $\mathrm{RBC} \mathrm{BH} 4$ content.

The question arises whether the permeability of the $\mathrm{RBC}$ membrane in younger animals is limited by $\mathrm{BH} 4$ biosynthesized intracellularly, or whether $\mathrm{BH} 4$ influx into the RBC may exceed BH4 efflux, especially in the neonatal Wistar rat. The former assumption is not compatible with the finding that the developmental changes in the permeability coefficient are not observed while the RBC BH4 content changed dramatically. The latter assumption, that $\mathrm{BH} 4$ accumulates in the cells by a flux mechanism, was also not consistent with the in vitro experiments which demonstrated that rat RBCs appeared to be more permeable for efflux than influx of $\mathrm{BH} 4$ (Table 5).

Phenylalanine is another factor which may influence $\mathrm{RBC}$ and plasma $\mathrm{BH} 4$ levels. Previous investigations have demonstrated that plasma BH4 was elevated by a phenylalanine load, both in humans $(12,18)$ and rats $(21)$. However, in the present study, plasma phenylalanine levels did not differ among the age groups either in humans or Wistar rats, while the phenylalanine level was slightly higher in human cord blood than other age groups (Table 6). Thus, regulation of $\mathrm{BH} 4$ biosynthesis by phenylalanine could not explain the higher BH4 levels in neonatal rat RBCs. 
Factors other than those examined appear to be responsible for maintaining higher RBC BH4 levels in younger rats. For folate, another pterin in animals, an $\mathrm{RBC}$ binding protein has been suggested as an important regulator of uptake into the cells, because the binding capacity of RBCs was increased in folate-deficient rats (22). If a specific BH4-binding protein also exists in plasma or cells, intracellular BH4 levels could be regulated by such a binding protein. In our preliminary studies, the majority of $\mathrm{BH} 4$ in human plasma was found in the protein fraction, rather than existing in the unbound form (data not shown). Since only unbound $\mathrm{BH} 4$ could penetrate into the cells, the RBC may function as a reservoir for unbound $\mathrm{BH} 4$ in the circulation, especially when excess $\mathrm{BH} 4$ exists.

In conclusion, the present study demonstrated that the BH4 dynamics in RBC were significantly different among species. In contrast to humans, rats showed remarkable developmental changes in the RBC BH4 content. However, these changes could not be simply explained by developmental changes in either the biosynthesizing activity or the permeability to $\mathrm{BH} 4$, or the plasma phenylalanine levels. Other factors which participate in maintaining cellular $\mathrm{BH} 4$, such as plasma and RBC BH4-binding may be involved.

We would like to thank all the hospital staff who helped us to collect the human blood samples. We would also like to express special appreciation to Ms. Y. Tabei and M. Imanishi for technical assistance. Finally, we deeply appreciate the critical reading of the manuscript by Dr. Philip D. Walson, Children's Hospital, Columbus, $\mathrm{OH}$.

\section{REFERENCES}

1) Duch, D. S., Bowers, S. W., Woolf, J. H., and Nichol, C. A. (1984): Biopterin cofactor biosynthesis. GTP cyclohydrolase, neopterin and biopterin in tissues and body fluids of mammalian species. Life Sci., 35, 1895-1901.

2) Andondonskaja-Renz, B., and Zeitler, H. J. (1983): Sepiapterin of pteridines from blood cells and plasma by reverse-phase high-performance liquid chromatography. Anal. Biochem., 133, 68-78.

3) Kaufman, S. (1959): Studies on the mechanism of the enzymatic conversion of phenylalanine to tyrosine. J. Biol. Chem., 234, 2677-2682.

4) Nagatsu, T., Levitt, M., and Udenfriend, S. (1964): Tyrosine hydroxylase. The initial step in norepinephrine biosynthesis. J. Biol. Chem., 234, 2677-2682.

5) Friedman, P. A., Kappleman, S., and Kaufman, S. (1972): Partial purification and characterization of tryptophan hydroxylase from rabbit hind brain. J. Biol. Chem., 247, 4165-4173.

6) Tayeh, M. A., and Marletta, M. A. (1989): Macrophage oxidation of L-arginine to nitric oxide, nitrite, and nitrate. J. Biol. Chem., 264, 19654-19658.

7) Kwon, N. S., Nathan, C. F., and Stuehen, D. J. (1989): Reduced biopterin as a cofactor in the generation of nitrogen oxides by murine macrophages. J. Biol. Chem., 264, 20496-20501.

8) Curtius, H.-Ch., Muldner, H., and Niederwieser, A. (1982): Tetrahydrobiopterin; 
efficacy in endogeneous depression and Parkinson's disease. J. Neurol. Transm., 55, 301-308.

9) Narabayashi, H., Kondo, T., Sugimoto, T., and Matsuura, S. (1982): Tetrahydrobiopterin administration for parkinsonian symptoms. Proc. Jpn. Acad., 58, 283-287.

10) Curtius, H. Ch., Niederwiser, A., Levine, R. A., Lovenberg, W., Woggon, B., and Angst, J. (1983): Successful treatment of depression with tetrahydrobiopterin. Lancet, i, 657-658.

11) Kaufman, S., Kapatos, G., McInns, R. R., Shulman, J. D., and Rizzo, W. B. (1982): Use of tetrahydropterins in the treatment of hyperphenylalaninemia due to defective synthesis of tetrahydrobiopterin. Evidence that peripherally administered tetrahydropterins enter the brain. Pediatrics, 70, 376-380.

12) Ponzone, A., Guardamagna, O., Spada, M., Ponzone, R., Sartore, M., Kierat, L., Heizmann, C. W., and Blau, N. (1993): Hyperphenylalaninenemia and pterin metabolism in serum and erythrocytes. Clin. Chim. Acta., 216, 63-71.

13) Hoshiga, M., Hatakeyama, K., Watanabe, M., Shimada, M., and Kagamiyama, H. (1993): Autographic distribution of [14C] tetrahydrobiopterin and its developmental change in mice. J. Pharm. Exp. Ther., 267, 971-978.

14) Kapatos, G., and Kaufman, S. (1981): Peripherally administered reduced pterins do enter the brain. Science, 212, 955-956.

15) Shoedon, G., Curtius, H.-Ch., and Niederwieser, A. (1987): Localization of GTP cyclohydrolase $I$ in human peripheral blood smears using a specific monoclonal antibody and an immune-alkaline phosphatase labeling technique. Biochem. Biophys. Res. Commun., 148, 1232-1236.

16) Blau, N., and Niederwieser, A. (1985): GTP cyclohydrolase; a review. J. Clin. Chem. Clin. Biochem., 23, 169-176.

17) Firgaira, F. A., Cotton, R. G. H., and Danks, D. M. (1979): Dihydropteridine reductase deficiency diagnosed by assays on peripheral blood-cells. Lancet, ii, 12601263.

18) Leeming, R. J., Hall, S. K., Surplice, I. M., and Green, A. (1990): Relationship between plasma and red cell biopterins in acute and chronic hyperphenylalaninemia. $J$. Inherited Metab. Dis., 13, 883-887.

19) Fukushima, T., and Nixon, J. C. (1980): Analysis of reduced forms of biopterin in biological tissues and fluids. Anal. Biochem., 102, 176-188.

20) Bradford, M. M. (1976): A rapid and sensitive method for the quantitation of microgram quantities of protein utilizing the principle of protein-dye binding. Anal. Biochem., 72, 248-254.

21) Dhondt, J.-L., Ardouin, P., Hayte, J.-M., and Farriaux, J.-P. (1981): Developmental aspects of pteridine metabolism and relationship with phenylalanine metabolism. Clin. Chim. Acta., 116, 143-152. 